\title{
Evaluating Mental Health First Aid: an Evidence Synthesis Review of Published Empirical Studies.
}

Opeyemi Atanda ( $\sim$ atandao2@lsbu.ac.uk)

London South Bank University https://orcid.org/0000-0001-8820-6596

Patrick Callaghan

London South Bank University School of Applied Sciences

Eleni Vangeli

London South Bank University School of Applied Sciences

Paula Reavey

London South Bank University School of Applied Sciences

Tim Carter

University of Nottingham

Steven D. Brown

Nottingham Trent University

Graham Durcan

Centre for Mental Health

Nick O'Shea

Centre for Mental Health

Sarah White

St George's University of London Division of Population Health Sciences and Education: St George's University of London Population Health Research Institute

Kerry Wood

London South Bank University School of Applied Sciences

\section{Research}

Keywords: Mental health first aid, evaluations, reviews, mixed methods studies, mental health literacy

Posted Date: December 11th, 2020

DOI: https://doi.org/10.21203/rs.3.rs-124268/v1

License: (c) (i) This work is licensed under a Creative Commons Attribution 4.0 International License. Read Full License 


\section{Abstract}

Background: Mental Health First Aid (MHFA) has received substantial international attention since its founding in the late 1990s, with a growing evidence base relating to its nature and impact across a variety of settings.

Aims: To identify the effectiveness of MHFA upon a range of outcomes, recipients, its cost-effectiveness, and the mechanisms of its effect.

Method: A systematic evidence synthesis

Results: Data from 65 studies show MHFA education improves trainees' mental health literacy, their perceived confidence in helping people living with mental distress and their intentions to help such people. MHFA also raises employees' knowledge of mental illnesses in the workplace. There was also evidence of MHFA trainees using aspects of the five-stage ALGEE MHFA approach in their helping behaviour. The quality of the studies in this review varied across different types of studies. No published studies to date have evaluated MHFA's impact on recipient outcomes, articulated the mechanisms of its effect, its cost-effectiveness, or societal impact.

Conclusions: MHFA remains popular, but evidence of its effectiveness upon those receiving it remains unknown. It is urgent to undertake studies testing the effectiveness and cost-effectiveness of MHFA upon recipients, as well as identifying, empirically, how MHFA works, for whom, under what conditions, and barriers to its implementation. Given that the enthusiasm and acceptance of MHFA appears widespread, systematic evaluations of its social impact are warranted.

Registration: The review protocol has been submitted to the Open Science Framework (View-only link: https://osf.io/rj4uh/? view_only=d1f9f2ed73724b3f8075c0c4581d0d87).

\section{Contributions To The Literature}

- In this paper, we report on the effectiveness of Mental Health First Aid (MHFA) upon a range of outcomes and the mechanisms of its effect. This would contribute to the current literature on complex intervention aimed at supporting individuals struggling with a mental health challenge.

- We are also contributing to the current literature on the role of theory in intervention design and its relevance to implementation research.

- We also understand that Systematic Reviews provides a unique home for evidence synthesis of the process by which effects are achieved. This review describes the mechanisms of the effect of MHFA.

\section{Background}

The concept of mental health literacy was developed by Jorm et al. (1997) who identified the need to improve the mental health literacy of the public. This concept gave birth to the rise of mental health first aid (MHFA): "Knowledge and beliefs about mental disorders that aid their recognition, management, or prevention" (Jorm et al., 1997, p. 182). MHFA is thus a mental health literacy intervention designed to equip trainees with the necessary skills to help individuals experiencing mental distress access appropriate help. The stages of a MHFA intervention are shown in Fig. 1.

Following the establishment of MHFA in Australia in 2000, it has received national support across countries where it's been established. MHFA has been implemented to engage UK employers in mental health awareness (Harvey et al., 2014). The cost of managing mental ill-health has strong links to companies' productivity and levels of absenteeism; the worldwide figure accounts for the estimated cost to the global economy of US\$1 trillion per year in lost productivity as a result of reported anxiety and depression (WHO / Mental Health in the Workplace, 2019). Recent UK figures estimate 15.4 million working days were lost in 2017/18 as a result of mental health problems, up from 12.5 million the previous year (Wison, 2018).

The aim of the current review was to appraise all published empirical literature on MHFA to understand the impact of MHFA on recipients, trainees, Mental Health First-Aiders, companies adopting MHFA and, where possible, the wider societal impact using a realist line of enquiry. The review sought to 1) determine the effectiveness of MHFA, 2) identify the mechanisms of its success and barriers to MHFA; 3 ) understand the influence of context on reported outcomes across all current knowledge; 4) develop an evidence-based recommendation to support future studies based on identified gaps in literature.

\section{Methods}

\section{Study Identification}

An initial search of "mental health first aid" through CINAHL yielded 211 articles. The search was expanded using 4 main electronic databases (CINAHL, MEDLINE, PSYCHINFO, PSYCHARTICLES) with search terms including "Mental health first aid" or "mental health literacy intervention" and "qualitative study" and "evaluation" and "systematic review" or "meta-analysis" .Searches were also conducted on Clinicaltrials.gov, MHFA England website, MHFA Australia and MHFA USA Website. All articles considered to have met the inclusion criteria outlined below were included. To ensure that studies were not omitted, the final list of included studies was crossed checked against the summary of all studies listed in the database for MHFA England, MHFA Australia, and MHFA USA.

\section{Eligibility criteria}

From an initial review of the literature, reviewers agreed the following criteria. 
1. Empirical studies assessing the impact of MHFA and (or) MHFA Training.

2. Reviews of empirical studies (meta-analytic, meta-synthesis or narrative).

3. Qualitative and quantitative studies and descriptive empirical studies of MHFA.

4. Empirical studies that have used any other version of MHFA (Youth MHFA, MHFA-Armed Forces, Teen MHFA as a mental health literacy intervention).

\section{Exclusion criteria}

1. Empirical studies that have used MHFA combined with other interventions where it was impossible to detect the effect of MHFA alone.

2. Literature not available in the English language.

3. Studies covered in the systematic reviews and meta-analyses.

4. All pilot or feasibility studies of MHFA.

5. All studies that have evaluated MHFA as part of a range of mental health literacy interventions (i.e., all other interventions that have used MHFA as a foundation against the standard courses of Adult MHFA 12hr, YMHFA 8hr)

\section{Screening}

Titles and abstracts were screened by one reviewer $(\mathrm{OA})$ for initial eligibility. Full texts were then reviewed by one reviewer (OA) to confirm eligibility. Where articles fell outside the inclusion criteria, the papers were additionally reviewed by two independent reviewers (PC) and (KVW), and the decision was taken to either include or exclude through consensus.

\section{Data extraction}

Following the guidelines suggested by the Centre for Reviews and Dissemination (CRD) (2009), one reviewer (OA) extracted data using a tailored version of a standardized form. All extracted data were subject to cross checking by an independent reviewer (PC or EV).

The following data were extracted for each included study:

- Author, year of publication and country

- Aims of the study

- Participant characteristics

- Study characteristics/contexts

- Outcome measures (quantitative studies or the quantitative element of studies using mixed methods where possible)

- Themes (qualitative \& the qualitative component of studies using mixed methods where possible)

- Expert eligibility (Delphi studies)

\section{Quality Assessment.}

All quantitative, qualitative, and mixed method studies were quality-assessed using a modified tool appropriate for mixed-studies review (Mixed Methods Appraisal Tool; MMAT) (Hong et al., 2018). The systematic reviews were quality checked against the PRISMA statement and checklist; the Delphi studies were quality-assessed based on the quality criteria proposed by Diamond et al. (2014). Details of the MMAT tool are shown in table 1. Details of the quality assessment of all studies included have been well-documented in the appendix.

\section{Data Synthesis}

Due to the variety of study designs in the included studies in this review, the data extracted were descriptively summarized. We have organized the information based on each methodological category, extracting the outcomes, and linking them to the mechanisms and the impact of the various contexts in which MHFA is used.

\section{Results}

Of the 226 papers assessed, following removal of duplicates, 66 met the inclusion criteria under the following categories: Review, Systematic Reviews \& MetaAnalyses ( $n=4)$, Qualitative studies ( $n=9)$, Quantitative Non-Randomized studies ( $n=20)$, Quantitative Randomized studies ( $n=3)$, Mixed method studies ( $n=8)$, Delphi Studies $(n=20)$, and other studies $(n=2)$. Details of search results are included in the supplementary file of this review.

\section{Systematic reviews and Meta-analyses}

Two meta-analyses (Hadlaczky et. al., 2014)(Maslowski et. al., 2019), a combined systematic review \& meta-analysis (Morgan et. al., 2018) and a review (Bell et. al., 2018) were included in the review. Using the PRISMA checklist (Preferred Reporting Items for Systematic Reviews and Meta-Analyses), the details of each review's compliance are detailed in table 2 .

Hadlaczky et al. (2014) assessed 15 studies evaluating MHFA training delivered over a 9 or 12-hour period using meta-analysis. The samples typically consisted of a self-nominated population. As reported in this review, MHFA training improved trainees' mental health knowledge and reduced participants' negative attitudes with a combined mean effect size of $0.56(95 \% \mathrm{Cl}=0.38-0.74 ; \mathrm{p}<0.001)$ for the identification of mental health problems and trainees' the 
knowledge about effective treatment. The observed differences in negative attitudes pre \& post measures was reported as moderate $($ effect size $=0.28$ ( $95 \%$ $\mathrm{Cl}=0.22-0.35 ; \mathrm{p}<0.001)$. While MHFA training was also reported to be effective in increasing trainees' 'help-providing' behavior with a reported small effect size $(0.25,95 \% \mathrm{Cl}=0.12-0.38 ; \mathrm{p}<0.001)$ this was based on the opportunities that trainees had to provide help, rather than actual help.

Morgan et al. (2018) identified 18 studies (4 cluster-RCTs, 10 RCTs, and 4 controlled trials), six were included in the previous meta-analysis by Hadlaczky and colleagues (2014) which were 4 controlled trials and 2 RCTs, the others were excluded because they were only single-group pre/post studies. Like the studies included in Hadlaczky's meta-analysis, most were conducted in Australia $(n=8)$, while others were conducted across North America $(n=5)$, Europe/UK ( $n=4)$ and Hong Kong $(n=1)$

In line with Hadlaczky et al., (2014), Morgan \& colleagues (2018) reported an improvement in trainees' knowledge, the study interpreted their effect sizes based on Cohen's work (Cohen, 1992) and they have reported a high effect size at post-intervention $(d=0.72(95 \% \mathrm{Cl}=0.59,0.86 ; \mathrm{p}<0.001)$, which was smaller (moderate based on Cohen's guideline) at 6-month follow-up ( $d=0.54(95 \% \mathrm{Cl}=0.43,0.64 ; \mathrm{p}<0.001)$ and 12 month follow-up ( $d=0.31(95 \% \mathrm{Cl}=0.09,0.53 ; p<$ 0.006). Small effects were reported for stigmatizing attitudes across all post intervention periods. Further analyses were conducted exploring the type of stigma, most included measures for social distance and personal stigma. Additionally, the perceived confidence levels of mental health first aiders have been consistently reported to significantly improve post-intervention $(0.58(95 \% \mathrm{Cl}=0.29,0.87 ; \mathrm{p}<0.001) \& 0.46(95 \% \mathrm{Cl}=0.31,0.62 ; \mathrm{p}<0.001)$ at 6 -months followup (Morgan et al., 2018). The effect sizes for trainees' intentions to provide MHFA post-intervention were moderate $0.75(95 \% \mathrm{Cl}=0.60,0.91 ; \mathrm{p}<0.001)$, and $0.55(95 \% \mathrm{Cl}=-0.08,1.18 ; \mathrm{p} .085)$ at 6 -months and a smaller effect size was reported $(0.2695 \% \mathrm{Cl}=-0.12,0.64 ; \mathrm{p} .182)$ at $12 \mathrm{months}$ follow-up.

Furthermore, there was not a statistically improvement post-intervention in the amount of help provided following MHFA training, but small improvements of $0.23(95 \% \mathrm{Cl}=0.08,0.38: \mathrm{p} .002)$ at 6-months post-intervention. In addition, no statistically significant improvement was reported in trainees' and recipients' mental health accessed via trainees of the intervention compared to baseline, pre-intervention (Morgan et al., 2018).

Maslowski et al. (2019) meta-analysis reported a similar outcome to Morgan's' (2018) with additional outcomes for the distress levels of trainees and 'recipient outcomes'. Effect sizes were reported using Hedges' g (Freeman et al., 1986), which was considered based on its precise estimate when sample sizes are small. Effect size magnitude was interpreted based on the standards of small (0.20-0.49), medium (0.50-0.79) and large (0.80 or greater) which is similar to Cohen's guidelines. The effect size for trainees' knowledge was moderate $(0.53,95 \% \mathrm{Cl}=0.39,0.66 ; \mathrm{p}<0.001)$, which was similar to the outcomes reported by Hadlaczky et al. (2014), whereas Morgan et al. (2018) reported effect sizes ranging from 0.22 to 0.72 for trainees' knowledge and recognition of recipients' mental health issues at post-intervention, and 6-months respectively. Regarding the attitudinal changes of trainees, the results reported were consistent with Morgan's with a small effect of 0.18 . Moreover, the review reported a moderate effect size $0.50(95 \% \mathrm{Cl}=0.34,0.67 ; \mathrm{p}<0.001)$, relative to that from Hadlaczky et al. $(2014 ; 0.25)$ for the combined self-reported behavioural and confidence outcomes for the trainees of MHFA.

Recipients in the studies Maslowskis' reviewed were not direct recipients. Two studies (Jorm et al., 2010 ; Lipson et. al., 2014) included in Maslowski's metaanalysis observed outcomes in a general population where MHFA was introduced. Lipson \& colleagues (2014) surveyed residents of a university hall of residence, while current students of teachers trained on MHFA in a school year were surveyed by Jorm \& colleagues (2010). Based on the review, Maslowskis reports, there were no reported effects on recipient's outcome which was observed based on changes in psychological distress of the recipients.

A evidence synthesis review carried out by Bell \& colleagues (Bell et al., 2018) investigated the application of MHFA in workplace settings. The review included 22 studies with the intention to answer the three research questions which investigated if there been an increase in awareness of mental health amongst employees (i.e. all staff employed by an organisation, including leaders/managers) receiving MHFA training; if there is any evidence of improved management of mental health in the workplace as a consequence of the introduction of MHFA training; and if there are evidences that the content of the MHFA training has been considered for workplace settings. They included the following study design; 13 single studies, 3 reviews, 3 single study protocol and 3 Delphi studies. In line with the previously reported systematic review \& meta-analyses, the Bell et al review reported consistent evidence that MHFA raises employees' knowledge of mental illnesses. MHFA trainees were more aware of where to find information and professional support and have shown increased confidence to render help to individuals experiencing a mental health crisis. The review also highlighted the lack of evidence from the published studies about the impact of introduction of MHFA in workplaces on the support provided by those trained and the sustainability of such actions of support. In addition, Bell et al reported limited evidence to on the adaptability of MHFA to different workplaces.

In sum, all the review studies included in this section reported on trainee related outcomes which showed an improvement of moderate or high at different time-points. Most significant trainee outcomes reported were knowledge of mental health, confidence of trainees to help someone struggling with a mental health crisis, trainees' attitudes was also reported with closer attention placed on different forms of stigma and the trainees psychological distress as a result of the MHFA training. Whereas the effect on the so-called recipients ranged from none too small with the reviews reporting recipient related outcomes around changes in the psychological distress which were all indirectly observed via trainees. However, none of the reviews found studies that gathered data from actual recipients of MHFA.

\section{Quantitative Non-Randomized \& Randomized studies}

Twenty-three studies are reviewed in this section, the detailed characteristics of the studies \& their quality checks are shown in Table $3 \& 4$.

Fifteen (65\%) of the included studies were non-randomized cohort studies that adopted the use of either a pre \& post study or time series design (pre, post, and follow-up design). Sample sizes range from $n=29$ (Borrill, 2010) to 606,941 (El-Amin et. al., 2018). The remaining included studies comprised 2 (9\%) quantitative non-randomized controlled trials, 2 (9\%) quantitative non-randomized cross-sectional studies, 1 (4\%) quantitative non-randomized descriptive study, and $3(13 \%)$ randomised control trials. 
Improvement in the perceived confidence levels of participants following MHFA training was generally reported. Boukouvalas et al. (2018) \& El-Den et al. (2018) observed changes in confidence levels using simulated patient case studies amongst university pharmacy students and the use of vignettes assessed with an ALGEE-based assessment rubic. These vignettes were reported to satisfy diagnostic criteria and correct recognition has been validated by a group of mental health professionals in Australia (Morgan et. al., 2013).

Hart et al. (2018) utilized a cluster-randomized crossover trial to assess the efficacy of the teen version of the MHFA intervention used in schools to encourage students' supportive behaviours towards their peers. The study also assessed, the quality of first aid intentions, recognition of mental health, beliefs about the helpfulness of adults and their measures of stigmatizing beliefs. Trainees were assessed against individuals who were also trained within the same study on a physical first aid course (PFA). All outcomes except the measures of stigmatizing beliefs were observed using a vignette with characters, John \& Jeanie presenting with suicidal ideation/depression and social anxiety/phobia, respectively. The vignette was developed based on teaching in the teenMHFA training

The study reported a medium effect size favouring teenMHFA ( $d s=0.50-0.58)$ based on a group-by-time interaction for the primary outcome. The primary outcomes, 'helpful first aid intentions' towards John/Jeanie, showed significant group-by-time interactions with medium effect sizes favoring tMHFA. In comparison to the PFA group, teenMHFA also reported a medium effect size and improvement in trainees' confidence supporting a peer (ds $=0.22-0.37$ ) and there was a medium effect size but small improvement in the number of adults rated as helpful ( $d s=0.45-0.46$ ). However, reductions in effect sizes were observed in stigmatizing beliefs ( $d s=0.12-0.40$ ) and 'harmful first aid intentions' towards John/Jeanie $(\mathrm{ds}=0.15-0.41)$.

Studies carried out by organizations in the UK via post-training questionnaires show that of 616 (88\%) of MHFA trainees who responded post-course, 34 (6\%) trainees) reported they used their MHFA skills when in contact with someone experiencing mental health distress (Brett-Jones, 2010). MacDonald et al. (2008) reported that 54 (85\%) of trainees who completed their follow-up questionnaire had reported offering help to individuals struggling with mental health issues. Heer et al. (2010) reported 100 (68.5\%) of their respondents had reportedly used the MHFA training to help someone with a mental health problem posttraining. Most of the respondents reported the type of help offered was listening (Heer, 2010; Macdonald et al., 2008) and giving assurances and information (Macdonald et al., 2010), two aspects of the ALGEE approach.

Three studies investigated the relationship between trainees' intention to provide help and the actual behavior of providing help in a bid to assess trainees' skills post-intervention (Rossetto et. al., 2014; Yap \& Jorm, 2012). Rossetto et al. (2014) investigated participants' responses to a vignette developed by the creators of MHFA (Kitchener \& Jorm, 2006) which was benchmarked against the ALGEE criteria. Responses were scored based on the level of details in each of the components of the action plan. However, the link between trainee's intention and their actual behaviour remains unclear. Methodology adopted by both studies is problematic in understanding the relationships between intentions and actual behaviour as the studies involved retrospective reporting of behaviour.

In Summary, there are limited studies evaluating the effects of MHFA training beyond a 6-month post intervention period, except a trial by Mohatt et al. (2017), and recent ones by Hart et al. (2018) and Reavley et al. (2018), indicating a need to measure the persistency of effects over a longer-term period. There has also been a lack of well-designed experimental, adequately powered studies (RCT or C-RCT) despite reviews (Hadlaczky et al., 2014; Maslowski et al., 2019; Morgan et al., 2019) identifying these as a consistent knowledge gap.

\section{Qualitative Studies}

Nine qualitative studies from Australia (4) and Wales (2), Hong Kong (1), Sweden (1), and the USA (1) are included in the review. Detailed characteristics of the studies \& their quality checks are shown in Table 5.

All included qualitative studies reported that part of the aim of conducting the studies was to explore the benefit of MHFA training for trainees. Improved confidence in relation to helping someone with a mental health condition was a common theme in the descriptive benefits of MHFA training across the studies. One Australian study (Jorm et. al., 2005), identified a positive perceived impact both on an intra-personal level emphasizing MHFA trainees' perceived empathy and confidence levels in providing help to others, and on an inter-personal level, focusing on how trainees manage crises.

A Swedish study (Svensson et al., 2015) reported participants referred to MHFA as a "toolbox" (p500) that increased their self-reported confidence to help someone with suspected mental distress. An Australian study using a case-study approach (Bovopoulos et. al., 2018) involving 14 participants reported more help-seeking behavior among workers which they reported as being associated with having MHFA trainees in the workplace, over and above that provided by Human Resources prior to the introduction of MHFA.

Two studies explored the experiences of Instructors in delivering MHFA training. Instructors interviewed in a Welsh study by Terry (2010) expressed the need for prerequisite mental health knowledge as an essential tool to enable them give relevant examples on how to identify mental health crises.

Undergraduate nursing student trainees of a MHFA training intervention in Hong Kong reported communication and interpersonal skills as key gains from MHFA training (Hung et. al., 2019). Other gains reported in a study by Pierce et al. (2010) are participants' perceived sense of empowerment to assist anyone who might be experiencing mental distress within their football club and their local community. An American study (Ploper et. al., 2015) reported that the presence of practical information in MHFA training and action plans developed in the training equipped trainees with skills to assist people experiencing mental distress, but there is no evidence of the assessment of the skills to which the authors allude.

Furthermore, Hung et al (2019) explored the impact of MHFA training on the trainees' awareness of their own mental health. Their findings suggest that MHFA training may enhance the personal awareness of the trainee's mental health as a secondary effect of enhancing their skills at helping others.

Bovopoulos et al. (2016) have also reported on MHFA instructors' experiences and perspectives of delivering MHFA courses in Australia. The main finding reported is that participants identified the need to adapt courses to suit the needs of first aiders from a range of cultural backgrounds and organizations. The 
influence of various organizational complexities on several outcomes needs to be further explored.

Bovopoulos et. al. (2018) explored the experiences of MHFA trainees or MHFA officers as the study calls them. Interviews were conducted across organizations that had previously adopted MHFA and appointed MHFA officers. Detailed analysis of participants responses reported the need for MHFA training to be offered to all staff in a bid to encourage a more acceptable atmosphere to have a mental health discussion within the workplace.

In sum, qualitative studies seek to describe, and explain participants' experiences of the issue's researchers' study, using themes 'emerging' from participants' narratives, and often drawing upon theoretical and conceptual insights as explanatory tools. But the data reported in the studies included here are largely impressionistic and provide little evidence about the impact of MHFA training on trainees' actual skills, especially in the application and impact of MHFA in 'real-world' settings where their skills are sought. The perceived gains in trainees' confidence, whether assessed by trainees or instructors, are arguably redundant in the absence of the application of the supposed confidence in practice, whether tested on the training, or in practice post-training. None of the included studies explored participants' impressions of the long-term effects of MHFA training on participants' confidence, the different ways participants implemented the interventions in their various settings, or possibilities and pitfalls implementing MHFA with recipients.

\section{Mixed Method Studies}

Eight studies were included three Australian, two American, two British, and one Canadian study. Details of the studies included are in Table 6 alongside details of their quality checks.

Massey et. al. (2014) reported MHFA training increased participants' knowledge of mental health conditions amongst those trained when compared with participants individuals in a control group under pre \& posttest conditions (effect size difference $=1.06$ ). In addition, Massey \& colleagues reported an increase in trainees' interactions with individuals with mental health conditions (Pretest $=75 \%$, Posttest $=86 \%$ ), recognizing more cases of mental health conditions (recognition of 2-4 people; pretest $58 \%$, posttest $80 \%$ ). However, a report of participants' openness towards people with a mental health condition, measured by vignette (Jorm et al., 2005) showed no difference from pre to posttest when compared with the those who did not receive training $(t(79)=-0.68, p>.05)$, However, participants in the qualitative component of the same study reported a perceived increase in their sensitivity and openness to people living with mental distress.

A similar mixed method study observed comparable results when MHFA training was delivered to coaches of a football team. The results reported by Massey et. al. (2014) have also been observed in a study by Talbot et al. (2017) showing that post-training, trainees reported that MHFA training helped in the promotion of treatment seeking as an indirect benefit of MHFA, though the study was based in a rural sector where trainees identified the lack of formal services which made it difficult to capture completed referrals based on contact with a mental health first aider.

Narayanasamy et al.'s feasibility study (2018) undertook a 3 investigation of the implementation, use and utility of MHFA in the workplace. Of all 139 respondents that filled out the survey, the study reported that the most common way which organizations have identified/selected trainees of MHFA training was via a general invitation sent out to employees with a selection criterion also implemented in some cases. Another common way was by direct request for training from employees whilst other organizations handpicked people from amongst their trainees. The study also reported on how trainees raised awareness of MHFA post-training; the most common strategy was the use of posters $72(51.8 \%)$; sharing of MHFA info about mental health \& wellbeing in the workplace 69 (49.6\%), running mental health days $63(45.3 \%)$ and intranet links 55 (39.6\%). The study also reported the mean effects of anticipated outcomes on the impact of MHFA in different workplaces was 28.07 which was higher than the actual mean effects (24.22) post-training based on feedback from their respondents Another cited issues such as the inclusion of more practical elements into MHFA education like more role-play sessions to rehearse the newly acquired skill from the training. On the other hand, 22 participants were interviewed across 6 organizations (2 public sector, 2 private sector \& 2 Not-for-profit sector). The aim of the interviews was to further explore the implementation \& use of MHFA. The study reported that having a clear rationale for introducing training, well-motivated MHFA coordinators and the existence of MHFA networks as important elements to successful implementation of MHFA in the workplace.

In sum, studies included in this section have explored the effect of MHFA using a mixed method approach. Similar to studies included in previous sections, there is a lack of longer follow-up period to observe the effects on MHFA across studies that explored the effects on trainees of MHFA (Massey et al., 2014; Narayanasamy et al., 2018). Retrospective rating of skills by trainees without the use of a validated survey instrument does not give a true picture of effects as adopted by Talbot \& colleagues (2017). Again, studies testing the impact of MHFA on recipients are lacking.

\section{DELPHI STUDIES}

The Delphi technique is known as a method of eliciting and refining group judgments (Dalkey, 1969). Studies using this methodology have been included in this review to understand how MHFA is administered to achieve its intended outcomes.

Following the development of MHFA, several problem-specific guidelines designed to enable mental health first aiders to better use the ALGEE approach were developed using Delphi- consensus methods. Fifteen studies are included here. The detailed characteristics of the studies \& their quality checks are shown in Table 7. While all included studies were conducted in Australia, the process of consensus was mostly international, with panels consisting of experts based in the USA, Ireland, UK, Canada, and New-Zealand.

This section has highlighted a range of guidelines on how to provide MHFA interventions to address specific issues ranging from mental health problems to other within different groups and communities. The development of these guidelines has been based on the main elements of MHFA (ALGEE) on how to support someone struggling with any form of mental health problem. However, the ALGEE steps have no scientifically established theoretical basis as they cannot be easily mapped against any evidence-based behaviour change taxonomies such as those developed by Michie et al. (2013). Furthermore, there is no 
empirical evidence to show the impact of the developed guidelines on their intended recipients. The impact of the guidelines on recipient outcomes remains a serious gap in the literature.

\section{Other studies}

A recently completed UK NIHR funded study - Wellbeing for Secondary Education (WISE), investigated two aspects of MHFA: the impact on teacher wellbeing, including effects on teachers' reports of depression, their sickness absence, and under-performance at work, and the impact on pupils' mental health and wellbeing. At the time of writing, there has been no published data from the main study, however, data from the pilot study shared by the authors reported an increase in confidence, knowledge, and self-reported helping behaviour amongst teachers, (Kidger et al., 2016), consistent with previous studies that have reported trainees outcomes (e.g.: Morgan et al., 2018)

\section{Discussion}

Following the introduction of MHFA in the late 1990s, it has been subject to a large body of empirical research investigating its application in varied settings. The purpose of this systematic evidence synthesis was to identify the effectiveness of MHFA upon its target audience, recipients, its cost-effectiveness, and its mechanisms of effect.

Context - The vast majority of the included studies were carried out in Australia, while others were spread across other countries with well-developed mental health services such as the UK, Canada, Wales, China, and Sweden amongst others. Evaluation studies have also developed alongside the growth of the intervention across a range of different contexts, with most of the studies carried out among various communities. Some studies have reported a 'call out' for volunteers as an approach to recruiting participants for their studies. This self-volunteering approach has seen more female participants than males being recruited onto courses. Another common characteristic across the participants are individuals with previous mental health knowledge or experiences. More controlled settings over recipient populations like educational institutions (primary-university settings), sports, and military settings have reported perceived impacts on service utilization and increased provision of help (Maslowski et al., 2019). However, there remains a lack of studies assessing the effects MHFA has on direct recipients. A comparison of direct recipients' outcomes in randomized controlled studies with active or passive controls is a notable absence in the literature.

Mechanism - By reviewing the range of identified mechanisms by which MHFA affects outcomes, we have identified a few mechanisms commonly related to trainee related outcomes, but only as perceived by trainees or their instructors. Participating in MHFA training improves mental health literacy and trainees' self-reported confidence levels (Maslowski et al., 2019). Furthermore, in relation to behavioural changes post-training regarding intention to help recipients or seek-help themselves, Rossetto \& colleagues reported a positive relationship between trainees intention scores and trainees behaviours to provide help which was similar to the findings reported by Yap \& Jorm (2012). This relationship only confirms the positive impact of MHFA training on the behaviours of trainees in providing help post-training (Jorm et al., 2005).

The operational definition of MHFA is the ALGEE approach. However, this 'model' lacks theoretical or conceptual clarity. There is considerable evidence of failings in studies assessing behavioural interventions to state clearly any theoretical model they have used to observe the constructs that underpin the intended behaviours observed (Kleinman \& Dougherty, 2013; Marcus et al., 2006; Michie et al., 2005),. Arguably, the primary purpose of MHFA is to improve mental health literacy, help people experiencing a mental health crisis and improve their help-seeking behaviour, whether it be self-treatment, professional, voluntary or other help. The latter two of these elements involve behaviour change on the part of mental health first aiders. Evidence shows that the most effective 'psychological interventions' are those underpinned by robust behaviour change theories (Michie et al., 2005). Currently, based on empirical evidence, MHFA, as defined by the ALGEE approach fails in this regard.

Outcomes - The reviews have emphasized quite a range of improvements in outcomes following MHFA, but these are focused largely on trainee-related outcomes such as mental health literacy, which includes knowledge and recognition of mental health problems. Other improvements include the perceived raising of the confidence levels of the trainees in rendering help to someone in distress or a mental health crisis and reducing stigmatizing attitudes towards people experiencing mental distress.

Studies have reported trainees' observation of positive effects of MHFA on recipients, but there is no direct or indirect impact of the intervention on recipients' actual outcomes (Maslowski et al., 2019). There have been no studies assessing the impact of this training on direct end-users of MHFA. Thus, well-designed studies of the impact of MHFA on recipient-specific outcomes are essential to improve the current evidence base.

Conducting a process evaluation which addresses a logic model to explain how Interventions like MHFA work is vital. Explaining these mechanisms could also help address critiques of MHFA (Defehr, 2016) as reproducing "psychocentrism" - the pathologizing of human problems that often arise from social inequalities rather than something people have.

\section{Strengths and limitations}

This is the first evidence synthesis investigating the effectiveness of MHFA across all of levels of involvement. Using rapid realist review methodology, we have included studies from a sizeable body of mostly published work which makes our findings are better than those we would have gotten from a conventional review of the effectiveness of MHFA. One limitation of this review is short time frame in which a rapid realist review of this sort are typically conducted which can make it difficult to fully theorize the mechanisms as well the linkages between context, mechanisms and outcomes as typically done in a realist review. This may consequently limit the generalizability and potency of findings. We could also attribute that limitation to the small amount of literature available to draw the proposed relationships. 


\section{Future work}

There is a pressing need for more and better-designed studies that evaluate MHFA against recipient outcomes. A better test of this surely would be reliably capturing the actual skills developed and used in real-world settings. The qualitative studies in this review has not helped in this regard. Evaluations of MHFA would be more informative and reliable if data are collected from a representative group of end-users. This would include end users from a variety of settings, for example: among the wider population in community studies, workplaces, among particular communities such as young people, older adults, the armed forces and include data on age, gender, and ethnicity, level of education, marital status, accommodation status, and nature and severity of presenting problems.

\section{Conclusions}

MHFA remains popular, but evidence of its effectiveness upon those receiving it remains unknown. It is urgent to undertake studies testing the effectiveness and cost-effectiveness of MHFA upon recipients, as well as identifying, empirically, how MHFA works, for whom, under what conditions, and barriers to its implementation. Given that the enthusiasm and acceptance of MHFA appears widespread, systematic evaluations of its social impact are warranted.

\section{Abbreviations}

MHFA: Mental health First Aid

YMHFA: Youth Mental Health First Aid

ALGEE: Approach the person, assess and assist with any crisis; Listen and communicate non-judgmentally; Give support and information; Encourage the person to get appropriate professional help and Encourage them to seek other forms of support.

MMAT: Mixed Methods Appraisal Tool

RCT: Randomised Controlled Trial

\section{Declarations}

\section{Ethics approval and consent to participate}

Not Applicable

\section{Consent for publication}

Not Applicable

\section{Availability of data and materials}

We have prior to this submission, submitted a review protocol for purpose of integrity documenting the process we have undertaken for this review unto the Open Science Framework (OSF). (View-only link: https://osf.io/rj4uh/?view_only=d1f9f2ed73724b3f8075c0c4581d0d87).

\section{Competing interests}

The authors declare they have no competing interests

\section{Funding}

No funding applicable

\section{Authors' contributions}

$\mathrm{PC}, \mathrm{PR}, \mathrm{EV}, \mathrm{KVW}$ and $\mathrm{OA}$ conceptualized the idea, OA drafted the manuscript. PC, PR, EV, KVW,TC provided the critical revision to the manuscripts. OA wrote the paper with input from all co-authors . PC \& KVW contributed to the inclusion and exclusion criteria. GD, SDB, SW and NOS reviewed the entire manuscripts and provided substantial comments for editing. All authors read and approved the final manuscript.

\section{Acknowledgements}

Not applicable

\section{References}

1. Articles included in the review

2. Bell, N., Evans, G., Beswick, A., \& Moore, A. (2018). Summary of the evidence on the effectiveness of Mental Health First Aid (MHFA) training in the workplace. RR135 Research Report. http://www.hse.gov.uk/research/rrhtm/rr1135.htm 
3. Bond, K. S., Jorm, A. F., Kelly, C. M., Kitchener, B. A., Morris, S. L., \& Mason, R. J. (2017). Considerations when providing mental health first aid to an LGBTIQ person: A Delphi study. Advances in Mental Health, 15(2), 183-197. https://doi.org/10.1080/18387357.2017.1279017

4. Borrill, J. (2010). Mental Health First Aid Training: Initial Evaluation by Private Sector Participants. August.

5. Boukouvalas, E. A., El-Den, S., Chen, T. F., Moles, R., Saini, B., Bell, A., O’Reilly, C. L., O'Reilly, C. L., \& O’Reilly, C. L. (2018). Confidence and attitudes of pharmacy students towards suicidal crises: patient simulation using people with a lived experience. Social Psychiatry \& Psychiatric Epidemiology, 53(11), 1185-1195. https://doi.org/10.1007/s00127-018-1582-2

6. Bovopoulos, N., LaMontagne, A. D., Martin, A., \& Jorm, A. F. (2018). Exploring the role of mental health first aid officers in workplaces: A qualitative study using case study methodology. International Journal of Workplace Health Management. https://doi.org/10.1108/IJWHM-06-2018-0082 LK http://bb2sz3ek3z.search.serialssolutions.com?sid=EMBASE\&issn=1753836X\&id=doi:10.1108\%2FIJWHM-06-20180082\&atitle=Exploring+the+role+of+mental+health+first+aid+officers+in+workplaces\%3A+A+qualitative+study+using+case+study+methodology\&stitle=In 381\&date

7. Bovopoulos, Nataly, Jorm, A. F., Bond, K. S., LaMontagne, A. D., Reavley, N. J., Kelly, C. M., Kitchener, B. A., \& Martin, A. (2016). Providing mental health first aid in the workplace: A Delphi consensus study. BMC Psychology, 4(1), 1-10. https://doi.org/10.1186/s40359-016-0148-x

8. Bovopoulos, Nataly, LaMontagne, A., Martin, A., \& Jorm, A. (2016). Delivering mental health first aid training in Australian workplaces: exploring instructors' experiences. International Journal of Mental Health Promotion, 18(2), 65-82. https://doi.org/10.1080/14623730.2015.1122658

9. Chalmers, K. J. K. J., Bond, K. S. K. S., Jorm, A. F. A. F., Kelly, C. M. C. M., Kitchener, B. A. B. A. B. A., Williams-Tchen, A. J. A. J., Chalmers, K. J. K. J., Bond, K. S. K. S., Jorm, A. F. A. F., Kelly, C. M. C. M., Kitchener, B. A. B. A. B. A., \& Williams-Tchen, A. J. A. J. (2014). Providing culturally appropriate mental health first aid to an Aboriginal or Torres Strait Islander adolescent: Development of expert consensus guidelines. International Journal of Mental Health Systems, 8(1), 6. https://doi.org/10.1186/1752-4458-8-6 LK - http://sfx.aub.aau.dk/sfxaub?sid=EMBASE\&issn=17524458\&id=doi:10.1186\%2F1752-44588 -

6\&atitle=Providing+culturally+appropriate+mental+health+first+aid+to+an+Aboriginal+or+Torres+Strait+Islander+adolescent\%3A+Development+of+exper \&date=2014\&auinit

10. Cohen, J. (1992). A power primer. Psychological Bulletin. https://doi.org/10.1037/0033-2909.112.1.155

11. Crone, D. M., Sarkar, M., Curran, T., Baker, C. M., Hill, D., Loughren, E. A., Dickson, T., \& Parker, A. (2019). Mental health first aid for the UK Armed Forces. Health Promotion International, 35(1), 132-139. https://doi.org/10.1093/heapro/day112

12. Dalkey, N. (1969). An experimental study of group opinion: The Delphi method. Futures. https://doi.org/10.1016/S0016-3287(69)80025-X

13. Department of Health and Social care, \& England, P. H. (2017). New $£ 15$ million programme to help train one million in mental health first aid - GOV.UK. In National Health Service. https://www.gov.uk/government/news/new-15-million-programme-to-help-train-mental-health-first-aiders

14. Diamond, I. R., Grant, R. C., Feldman, B. M., Pencharz, P. B., Ling, S. C., Moore, A. M., \& Wales, P. W. (2014). Defining consensus: A systematic review recommends methodologic criteria for reporting of Delphi studies. Journal of Clinical Epidemiology. https://doi.org/10.1016/j.jclinepi.2013.12.002

15. El-Amin, T., Anderson, B. L., Leider, J. P., Satorius, J., \& Knudson, A. (2018). Enhancing mental health literacy in rural America: Growth of Mental Health First Aid program in rural communities in the United States from 2008-2016. Journal of Rural Mental Health, 42(1), 20-31. https://doi.org/10.1037/rmh0000088

16. El-Den, S., Chen, T. F., Moles, R. J., O'Reilly, C., \& O'Reilly, C. (2018). Assessing mental health first aid skills using simulated patients. American Journal of Pharmaceutical Education, 82(2), 185-193. https://doi.org/10.5688/ajpe6222

17. Freeman, P. R., Hedges, L. V., \& Olkin, I. (1986). Statistical Methods for Meta-Analysis. Biometrics. https://doi.org/10.2307/2531069

18. Hadlaczky, G., Hökby, S., Mkrtchian, A., Carli, V., \& Wasserman, D. (2014). Mental health first aid is an effective public health intervention for improving knowledge, attitudes, and behaviour: A meta-analysis. International Review of Psychiatry, 26(4), 467-475.

https://doi.org/10.3109/09540261.2014.924910

19. Hart, L. M., Morgan, A. J., Rossetto, A., Kelly, C. M., Mackinnon, A., Jorm, A. F., L.M., H., A.J., M., A., R., C.M., K., A., M., \& A.F., J. (2018). Helping adolescents to better support their peers with a mental health problem: A cluster-randomised crossover trial of teen Mental Health First Aid. Australian and New Zealand Journal of Psychiatry, 52(7), 638-651. https://doi.org/10.1177/0004867417753552

20. Harvey, S., Joyce, S., Tan, L., Johnoson, A., Nguyen, H., Modini, M., \& Groth, M. (2014). Developing a mentally healthy workplace: A review of the literature. November, 73. http://citeseerx.ist.psu.edu/viewdoc/download?doi=10.1.1.682.5176\&rep=rep1\&type=pdf

21. Hong, Q. N., Fàbregues, S., Bartlett, G., Boardman, F., Cargo, M., Dagenais, P., Gagnon, M. P., Griffiths, F., Nicolau, B., O’Cathain, A., Rousseau, M. C., Vedel, I., \& Pluye, P. (2018). The Mixed Methods Appraisal Tool (MMAT) version 2018 for information professionals and researchers. Education for Information. https://doi.org/10.3233/EFI-180221

22. Hung, M. S. Y. Y., Lam, S. K. K. K., \& Chow, M. C. M. M. (2019). Nursing students' experiences of mental health first aid training: A qualitative descriptive study. Collegian, 26(5), 534-540. https://doi.org/10.1016/j.colegn.2019.02.006

23. Jorm, A. F. A. F., Kitchener, B. A. B. A., Sawyer, M. G. M. G., Scales, H., \& Cvetkovski, S. (2010). Mental health first aid training for high school teachers: A cluster randomized trial. BMC Psychiatry, 10. https://doi.org/10.1186/1471-244X-10-51 LK - http://sfx.aub.aau.dk/sfxaub?

sid=EMBASE\&issn=1471244X\&id=doi:10.1186\%2F1471-244X-10-

51 \&atitle=Mental+health+first+aid+training+for+high+school+teachers\%3A+A+cluster+randomized+trial\&stitle=BMC+Psychiatry\&title=BMC+Psychiatry\& \&date=2010\&auinit1 $=$ A\&auinitm $=F$

24. Jorm, A. F., Blewitt, K. A., Griffiths, K. M., Kitchener, B. A., \& Parslow, R. A. (2005). Mental health first aid responses of the public: Results from an Australian national survey. BMC Psychiatry, 5, 1-10. https://doi.org/10.1186/1471-244X-5-9

Page $9 / 12$ 
25. Jorm, A. F., Kitchener, B. A., \& Mugford, S. K. (2005). Experiences in applying skills learned in a Mental Health First Aid training course: A qualitative study of participants' stories. BMC Psychiatry, 5, 1-11. https://doi.org/10.1186/1471-244X-5-43

26. Jorm, A. F., Korten, A. E., Jacomb, P. A., Christensen, H., Rodgers, B., \& Pollitt, P. (1997). "Mental health literacy”: A survey of the public's ability to recognise mental disorders. Medical Journal of Australia, 166(4), 182-186. https://doi.org/10.5694/J.1326-5377.1997.TB140071.X

27. Kelly, C. M., Jorm, A. F., \& Kitchener, B. A. (2009). Development of mental health first aid guidelines for panic attacks: a Delphi study. BMC Psychiatry, 8 , 1-8. https://doi.org/10.1186/1471-244X-9-49

28. Kelly, C. M., Jorm, A. F., Kitchener, B. A., \& Langlands, R. L. (2008). Development of mental health first aid guidelines for suicidal ideation and behaviour: a Delphi study. BMC Psychiatry, 8, 17. https://doi.org/10.1186/1471-244X-8-17

29. Kidger, J., Stone, T., Tilling, K., Brockman, R., Campbell, R., Ford, T., Hollingworth, W., King, M., Araya, R., \& Gunnell, D. (2016). A pilot cluster randomised controlled trial of a support and training intervention to improve the mental health of secondary school teachers and students - the WISE (Wellbeing in Secondary Education) study. BMC Public Health, 16(1), 1060. https://doi.org/10.1186/s12889-016-3737-y

30. Kingston, A. H. A. H., Morgan, A. J., Jorm, A. F., Hal, K., Hart, L. M., Kelly, C. M., Lubman, D. I., A.J., M., A.F., J., K., H., L.M., H., \& C.M., K. (2011). Helping someone with problem drug use: A delphi consensus study of consumers, carers, and clinicians. BMC Psychiatry, $11,3$.

https://doi.org/http://dx.doi.org/10.1186/1471-244X-11-3

31. Kingston, A. H., Jorm, A. F., Kitchener, B. A., Hides, L., Kelly, C. M., Morgan, A. J., Hart, L. M., \& Lubman, D. I. (2009). Helping someone with problem drinking: Mental health first aid guidelines - a Delphi expert consensus study. BMC Psychiatry, 9, 1-7. https://doi.org/10.1186/1471-244X-9-79

32. Kitchener, B. A., Jorm, A. F., BA, K., \& AF, J. (2006). Mental health first aid training: Review of evaluation studies. Australian and New Zealand Journal of Psychiatry, 4O(1), 6-8. https://doi.org/10.1111/j.1440-1614.2006.01735.x

33. Kleinman, L. C., \& Dougherty, D. (2013). Assessing quality improvement in health care: Theory for practice. In Pediatrics. https://doi.org/10.1542/peds.2012-1427n

34. Langlands, R. L., Jorm, A. F., Kelly, C. M., \& Kitchener, B. A. (2008). First aid recommendations for psychosis: Using the Delphi method to gain consensus between mental health consumers, carers, and clinicians. Schizophrenia Bulletin, 34(3), 435-443. https://doi.org/10.1093/schbul/sbm099

35. Lipson, S. K., Speer, N., Brunwasser, S., Hahn, E., \& Eisenberg, D. (2014). Gatekeeper training and access to mental health care at universities and colleges. Journal of Adolescent Health, 55(5), 612-619. https://doi.org/10.1016/j.jadohealth.2014.05.009

36. Marcus, B. H., Williams, D. M., Dubbert, P. M., Sallis, J. F., King, A. C., Yancey, A. K., Franklin, B. A., Buchner, D., Daniels, S. R., \& Claytor, R. P. (2006). What we know and what we need to know: A scientific statement from the American Heart Association council on nutrition, physical activity, and metabolism (subcommittee on physical activity); council on cardiovascular disease in the young; and the interdis. In Circulation. https://doi.org/10.1161/CIRCULATIONAHA.106.179683

37. Maslowski, A. K., LaCaille, R. A., LaCaille, L. J., Reich, C. M., \& Klingner, J. (2019). Effectiveness of Mental Health First Aid: a meta-analysis. Mental Health Review Journal, 24(4), 245-261. https://doi.org/10.1108/MHRJ-05-2019-0016

38. Massey, J., Brooks, M., \& Burrow, J. (2014). Evaluating the effectiveness of mental health first aid training among student affairs staff at a Canadian university. Journal of Student Affairs Research and Practice, 51(3), 323-336. https://doi.org/10.1515/jsarp-2014-0032

39. Mendenhall, A. N., Jackson, S. C., \& Hase, S. (2013). Mental Health First Aid USA in a Rural Community: Perceived Impact on Knowledge, Attitudes, and Behavior. Social Work in Mental Health, 11(6), 563-577. https://doi.org/10.1080/15332985.2013.812542

40. MHFA Australia. (2017). \$2.45 Million Government Funding Boost for MHFA Australia / Mental Health First Aid. 2017. https://mhfa.com.au/news/201705-29/33203/245-million-government-funding-boost-mhfa-australia

41. Michie, S., Johnston, M., Abraham, C., Lawton, R., Parker, D., \& Walker, A. (2005). Making psychological theory useful for implementing evidence based practice: A consensus approach. Quality and Safety in Health Care. https://doi.org/10.1136/qshc.2004.011155

42. Michie, Susan, Richardson, M., Johnston, M., Abraham, C., Francis, J., Hardeman, W., Eccles, M. P., Cane, J., \& Wood, C. E. (2013). The behavior change technique taxonomy ( $v 1)$ of 93 hierarchically clustered techniques: Building an international consensus for the reporting of behavior change interventions. Annals of Behavioral Medicine. https://doi.org/10.1007/s12160-013-9486-6

43. Mohatt, N. V., Boeckmann, R., Winkel, N., Mohatt, D. F., \& Shore, J. (2017). Military Mental Health First Aid: Development and Preliminary Efficacy of a Community Training for Improving Knowledge, Attitudes, and Helping Behaviors. Military Medicine, 182(1), e1576-e1583. https://doi.org/10.7205/MILMED-D-16-00033

44. Morgan, A. J. A. J., Ross, A., Reavley, A. ., \& Reavley, N. J. (2018). Systematic review and meta-analysis of mental health first aid training: Effects on knowledge, stigma, and helping behaviour. PLOS ONE, 13(5), 1-20. https://doi.org/10.1371/journal.pone.0197102 LK - http://sfx.aub.aau.dk/sfxaub? sid=EMBASE\&issn=19326203\&id=doi:10.1371\%2Fjournal.pone.0197102\&atitle=Systematic+review+and+meta-

analysis+of+mental+health+first+aid+training\%3A+Effects+on+knowledge\%2C+stigma\%2C+and+helping+behaviour\&stitle=PLoS+ONE\&title=PLoS+ONE\& \&date=2018\&auinit1 $=A$ \&auinitm $=\mathrm{J}$

45. Morgan, A. J., Fischer, J.-A. A. A., Hart, L. M., Kelly, C. M., Kitchener, B. A., Reavley, N. J., Yap, M. B. H. H., Cvetkovski, S., \& Jorm, A. F. (2019). Does Mental Health First Aid training improve the mental health of aid recipients? The training for parents of teenagers randomised controlled trial. $B M C P s y c h i a t r y$, 19(1), 99. https://doi.org/10.1186/s12888-019-2085-8

46. Morgan, A. J., Jorm, A. F., \& Reavley, N. J. (2013). Beliefs of Australian health professionals about the helpfulness of interventions for mental disorders: Differences between professions and change over time. Australian and New Zealand Journal of Psychiatry. https://doi.org/10.1177/0004867413490035

47. Narayanasamy, M., Geraghty, J., Coole, C., \& Nouri, F. (2018). Mental health first aid in the workplace (MENTOR): A feasibility study. 1-99. www.iosh.co.uk/MHFAworkplace

Page $10 / 12$ 
48. National Council For Behavioral Health. (2014). SAMHSA 2014 Budget Includes $\$ 15$ Million for Mental Health First Aid - National Council. National Council For Behavioral Health . https://www.thenationalcouncil.org/press-releases/samhsa-2015-budget-includes-15-million-mental-health-first-aid/

49. Pierce, D., Liaw, S.-T. T., Dobell, J., \& Anderson, R. (2010). Australian rural football club leaders as mental health advocates: An investigation of the impact of the Coach the Coach project. International Journal of Mental Health Systems, 4, 1-8. https://doi.org/10.1186/1752-4458-4-10

50. Ploper, V., Jones, R., Kraus, D. J., Schmidt, A., \& Corrigan, P. (2015). Feedback from American participants of a mental health first aid training course. Journal of Public Mental Health, 14(2), 118-121. https://doi.org/10.1108/JPMH-04-2014-0016

51. Reavley, N. J., Morgan, A. J., Fischer, J.-A. A., Kitchener, B., Bovopoulos, N., \& Jorm, A. F. (2018). Effectiveness of eLearning and blended modes of delivery of Mental Health First Aid training in the workplace: randomised controlled trial. BMC Psychiatry, 18(1), 312. https://doi.org/10.1186/s12888-018-1888-3

52. Ross, A. M., Hart, L. M., Jorm, A. F., Kelly, C. M., \& Kitchener, B. A. (2012). Development of key messages for adolescents on providing basic mental health first aid to peers: A Delphi consensus study. Early Intervention in Psychiatry, 6(3), 229-238. https://doi.org/10.1111/j.1751-7893.2011.00331.x

53. Ross AM, Kelly CM, Jorm AF., Ross, A. M., Kelly, C. M., \& Jorm, A. F. (2014). Re-development of mental health first aid guidelines for non-suicidal self-injury: A Delphi study. BMC Psychiatry, 14(1), 1-8. https://doi.org/10.1186/s12888-014-0236-5

54. Rossetto, A., Jorm, A. F., \& Reavley, N. J. (2014). Examining predictors of help giving toward people with a mental illness: Results from a national survey of Australian adults. SAGE Open, 4(2). https://doi.org/10.1177/2158244014537502

55. Svensson, B., Hansson, L., \& Stjernswärd, S. (2015). Experiences of a Mental Health First Aid Training Program in Sweden: A Descriptive Qualitative Study. Community Mental Health Journal, 51(4), 497-503. https://doi.org/10.1007/s10597-015-9840-1

56. Talbot, J. A., Ziller, E. C., \& Szlosek, D. A. (2017). Mental Health First Aid in Rural Communities: Appropriateness and Outcomes. Journal of Rural Health, 33(1), 82-91. https://doi.org/10.1111/jrh.12173

57. Terry, J. (2010). Experiences of instructors delivering the Mental Health First Aid training programme: A descriptive qualitative study. Journal of Psychiatric and Mental Health Nursing, 17(7), 594-602. https://doi.org/10.1111/j.1365-2850.2010.01557.x

58. WHO / Mental health in the workplace. (2019). 2019. https://www.who.int/mental_health/in_the_workplace/en/

59. Wison, J. (2018). Work-related stress and mental illness now accounts for over half of work absences. The Telegraph. https://www.telegraph.co.uk/news/2018/11/01/work-related-stress-mental-illness-now-accounts-half-work-absences/

60. Yap, M. B. H., \& Jorm, A. F. (2012). Young people's mental health first aid intentions and beliefs prospectively predict their actions: Findings from an Australian National Survey of Youth. Psychiatry Research, 196(2-3), 315-319. https://doi.org/10.1016/j.psychres.2011.10.004

\section{Tables}

Due to technical limitations, tables 1 to 7 PDFs are only available as a download in the Supplemental Files section.

\section{Figures}

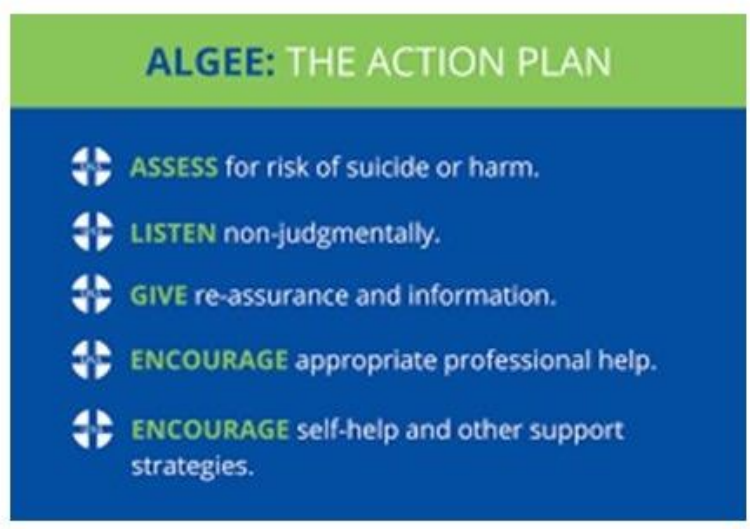

\section{Figure 1}

MHFA ACTION PLAN (ALGEE)

\section{Supplementary Files}

This is a list of supplementary files associated with this preprint. Click to download.

- Table1MixedMethodsAppraisalToolMMATversion2018.pdf

- Table2PRISMACHECKLISTFORSYSTEMATICREVIEWS.pdf

- Table3QUANTITATIVENONRANDOMIZEDSTUDIES.pdf

- Table4QUANTITATIVERANDOMIZEDSTUDIES.pdf 
- Table5QUALITATIVESTUDIES.pdf

- Table6MIXEDMETHODSTUDYCHARACTERISTICS.pdf

- Table7DELPHISTUDYXTERISTICSAGAINSTQUALITYCRITERIA.pdf

- AdditionalFilePRISMAFLOWDIAGRAM.pdf

- AdditionalfileQUALITYASSESSMENTSUMMARY.pdf

- PRISMAPchecklistEvidenceSynthesis.doc 